\title{
The Effect of Thoracic Joint Mobilization on the Changes of the Thoracic Kyphosis Angle and Static and Dynamic Balance
}

\author{
Hae-Jin Jeong ${ }^{*}$ and Byeong-Jo Kim ${ }^{\dagger ; * *}$ \\ Department of Biomedical Health Science, Dong Eui University, Busan 47340, Korea
}

\begin{abstract}
The objective of this study was to evaluate the effects of thoracic mobilization (TM) on the angle of thoracic kyphosis, and static and dynamic balances by application period. The subjects of this study were 18 adult males and females ( $\geq 20$ years old) who had the angle of thoracic kyphosis equal to or higher than $40^{\circ}$. A pre-test was conducted for all subjects and TM was carried out. Data were collected before the intervention, 3 weeks after the intervention, and 6 weeks after the intervention. It was measured three times per measurement and mean values were used for the analysis. The results of this study showed that the angle of thoracic kyphosis significantly $(P<.05)$ decreased after applying TM. However, the migration area $\left(\mathrm{mm}^{2}\right)$ of the center of pressure (COP) in the static balance did not vary significantly. In the case of the dynamic balance, when eyes were open, the migration area $\left(\mathrm{mm}^{2}\right)$ of the COP significantly $(P<.05)$ decreased after 3 weeks. When eyes were open, the migration area $\left(\mathrm{mm}^{2}\right)$ of the COP significantly $(P<.05)$ decreased after 3 weeks and 6 weeks. Therefore, an intervention for improving the human body alignment and balance should be applied for a long-term, rather than a short-term, in order to be effective.
\end{abstract}

Key Words: Thoracic kyphosis, Thoracic mobilization, Balance

\section{INTRODUCTION}

In recent years, the use of computer and information technology by the general public has been on the rise, and research findings indicate that South Korea has a smartphone penetration rate of 73 percent (Joo and Yoo, 2014). Excessive use of communications devices is a factor causing not only ophthalmic diseases, but also musculoskeletal disorders affecting areas such as the neck, shoulder, arm, and hand (Kim et al., 2016). In particular, it is known that young adults exposed continually and repeatedly to the frequent use of communications devices come to develop poor pos- ture habits, which can affect spinal diseases (Kim et al., 2018). The vertebral column is made up of 7 cervical vertebrae, 12 thoracic vertebrae and 5 lumbar vertebrae, and the vertebral canal is adjacent to the central and peripheral nervous systems. In addition, the spinal column produces its motion through the action of vertebrae, ligaments, muscles, and many joints (Kim, 2015). Casual poor postures practiced in everyday life lead to spinal transformation due to the change to ideal alignment of the body. As a result, the transformed loads imposed on the vertebrae increase the shearing force and compression force in the vertebra segment (Kim and Jang, 2012; Kim, 2013).

The curves of the sagittal plane of the vertebra come in the

Received: May 9, 2019 / Revised: June 19, 2019 / Accepted: June 20, 2019

* Graduate student, ${ }^{* *}$ Professor.

${ }^{\dagger}$ Corresponding author: Byeong-Jo Kim. Department of Biomedical Health Science, Dong-Eui University, 176 Eongwangno (gayadong san24), Busanjin-gu, Busan 47340, Korea.

Tel: +82-51-890-4221, Fax: +82-0505-182-6881, e-mail: pt123@deu.ac.kr

(C)The Korean Society for Biomedical Laboratory Sciences. All rights reserved.

(c) This is an Open Access article distributed under the terms of the Creative Commons Attribution Non-Commercial License (http://creativecommons.org/licenses/by-nc/3.0/) which permits unrestricted non-commercial use, distribution, and reproduction in any medium, provided the original work is properly cited. 
form of the lordotic curvature in the cervical vertebrae, the kyphosis of the thoracic vertebra and the lordotic curvature of the lumbar vertebrae. At birth, the primary curve in the form of retroversion is seen in the region of the thoracic vertebra and sacral vertebrae, and with growth, the secondary curves take place, which involve the lordotic curves being developed around the cervical vertebra and lumbar vertebrae. Keeping the balance in the sagittal plane involves the vertebra, pelvis, coxal articulation and knee joint (Lee et al., 2011). The vertebra has an S-shaped curve and supports a variety of physical activities by maintaining body alignment and postures (Mirbagheri et al., 2015).

An ideal body alignment line refers to a line through which all segments go through the axis of joints vertically from the gravity line. It refers to maintaining normal body alignment that connects to the front of knee and malleolus after passing the external auditory canal and shoulders, then the back of the vertex on the femoral head of the hip joint (Jung et al., 2013). Imbalance of the body due to changes in spinal alignment leads to the unnecessary use of postural muscles as a compensatory action, causing an excessive use of energy, fatigue and pain (Lee and Kang, 2016). Poor spinal alignment due to bad living habits, aging or injury causes changes in the pressure on the spinal disc and can change to anaplasia, along with vertebral deformation and disorders (Han et al., 2012). The thoracic vertebrae form ribs and joints making minor movements, but are very clinically important as the nerves which move from each joint to the trunk and abdominal viscera pass through them (Lee, 2010). They also play an important role in delivering arm and leg strength as the optimal functional segment that requires both stability and mobility (Jang et al., 2012).

The angle of thoracic kyphosis in the vertebral column involves the vertebral body and vertebral disc staying generally in the $20 \sim 40^{\circ}$ shape on the sagittal plane (Kim and Jang, 2012). When the retroversion around the thoracic vertebra exceeds its normal range, the lordotic curve is lost and the retroversion of thoracic vertebra is $40^{\circ}$ or over, a phenomenon called 'kyphosis' (Song et al., 2013). Kyphosis of the thoracic vertebrae is developed not by self-regulated vertebrae disorders but by the structural deformation of the thoracic vertebrae due to poor posture habits on a daily basis, as well as a decrease of facet joint movement. Therefore, the human body, once it detects a region where movements are decreased, causes overuse and hypermobility (Kim and Jang, 2012).

Keeping one's head and neck in a correct posture is possible only when the thoracic vertebrae are aligned in a correct manner (Jung et al., 2013). When a movement is sensed on a certain body segment by the interaction between spine, pelvis, knee and ankle by the influence of a lifestyle and environment, other segments show movements in response to it (Moon et al., 2014). Kyphosis is influenced by the weight load and movement patterns, and the greater level of stiffening of the thoracic vertebrae causes an incorrect posture control in the lumbar vertebrae and cervical vertebrae as a compensatory action (Song et al., 2013; Jung, 2015). The variation of the kyphosis influences movement patterns and makes compensatory movements in the neckbone and lumbar vertebrae, resulting in pain and functional disorder around the vertebrae (Kim and Jang, 2012).

Spinal joint mobilization is a method used to strengthen unstable joints by increasing the mobility range of joints that have limited movements due to changes in the musculoskeletal system caused by kyphosis and functional limitations across the body and weakened muscles. This treatment technique is found to have fewer side effects when applied indirectly to the neck or waist, rather than a direct application (Yang and Kim, 2015). When indirect joint mobilization was applied to the upper thoracic region of a patient with neck pain, it presented effects of a reduction in neck pain (Hwangbo, 2011).

Joint mobilization is classified into three phases depending on its purposes, and involves the restoration of joint mobility around the joints with minor functional movements as well as pain relief. The first and second phases are pulling and gliding, respectively, and aim at relieving pain, while the third phase - pulling and gliding - is used to increase joint mobility of stiff tissues with minor movements (Kaltenborn, 1993).

As such, earlier studies both in Korea and abroad have demonstrated that joint mobilization and correction exercise play a positive role in changes to kyphotic angle, the bending of neck bone and lumbar vertebrae and to pain. However, more studies need to be conducted to address the question 
of how the changes to the thoracic kyphotic angle during the application of joint mobilization to people with kyphosis influence the balance of the body.

As such, this study intended to identify the changes to the thoracic kyphotic angle that occur with the application of joint mobilization to people with a thoracic kyphosis greater than $40^{\circ}$, along with their impacts on static and dynamic balance.

\section{MATERIALS AND METHODS}

\section{Study design and study participants}

This study applied joint mobilization to all participants following the pretest three times each for six weeks, and it was measured a total of three times - before intervention, three weeks after intervention and six weeks after intervention. Each measurement was conducted three times and mean values were retrieved and used. Approved by the Bioethics Committee (DIRB-201709-HR-E-037) to ensure the rights and well-being of the participants and comply with the Act on Ethics and Safety of the Research. The participants were 18 male and female adults (5 males and 13 females) who agreed to take part in the study, with an average age range of $30.78 \pm 4.08$, height range of $163.22 \pm 5.35 \mathrm{~cm}$ and weight range of $61.78 \pm 12.46 \mathrm{~kg}$. The selected participants: 1) had a kyphosis angle of $40^{\circ}$ or over, 2) were male and female adults aged 20 or over, 3 ) had never undergone surgical treatment for vertebrae, 4) had no rheumatic diseases or arthritis in the spine, 5) had no nervous system disorders, and 6) had no other diseases that could affect the experiment.

\section{Experimental procedures and methods}

For a warm-up exercise prior to the application of the joint mobilization, the participants took a five-minute walk on a treadmill to promote temperature and blood circulation throughout their body. For a cool-down exercise, they walked on the treadmill for an additional five minutes (Song et al., 2013).

The therapist, standing at the patient's side, selected the segment with minor mobility from zygapophysial joints and let the lateral surface of the luna in the pisiform and hamate on the right hand come into contact with the spinous process on the spinal segment to be used. The therapist folded the left hand on the right hand to secure accurate support in the contact point and prevent shaking. While loosening the grip of the right hand, and allowing the left hand grip to tighten, movement was made to put the therapist's weight in line with the vertical axis. Then, instead of arm movement, the upper body was involved into top-to-bottom vibration motions so the patient's spinous process of vertebrae could be led to making back-to-front movements. In this way, the strength was transferred (Hyung and Ha, 2009; Sung et al., 2014). In terms of the amount of strength, this experiment applied Kaltenborn's three-phase mobilization techniques that generate an extensibility in articular tissues (Kaltenborn, 1993; Yang and Kim, 2015).

The participants were asked if they felt uncomfortable with the applied segment for the joint mobilization. This was conducted repeatedly three times after about one-minute application \& about one-minute break, while the patient was feeling comfortable (Hyung and Ha, 2009).

\section{Measurement instrument and methods}

The kyphosis angle was measured using two inclinometers (Lewis and Valentine, 2010). The kyphosis angle was calculated by the summation of the angle recorded by the two inclinometers (Fig. 1).

One horizontal section of the floor of the inclinometer is located between spinous processes in thoracic vertebrae 1 and 2, with the other placed between thoracic vertebra 12 and lumbar vertebra 1 . Prior to taking the measurement, the patient performed the following actions: taking off shoes, standing up straight, taking wide-open arm exercise three times, bending head forward and lifting three times, and breathing in \& out three times.

The Biorescue (RM Ingenierie, France), a measuring instrument used to measure balance ability, is designed to measure the movement of the body's center of pressure (COP) at a point where the gravity influences the center of gravity (COG) of the body or object in order to assess the migration area $\left(\mathrm{mm}^{2}\right)$ while the movement is taking place (Nam, 2015). The intra-class correlation coefficient (ICC) was in the $0.83 \sim 0.95$ range in the reliability analysis in the previous research (Geronimi, 2014). 

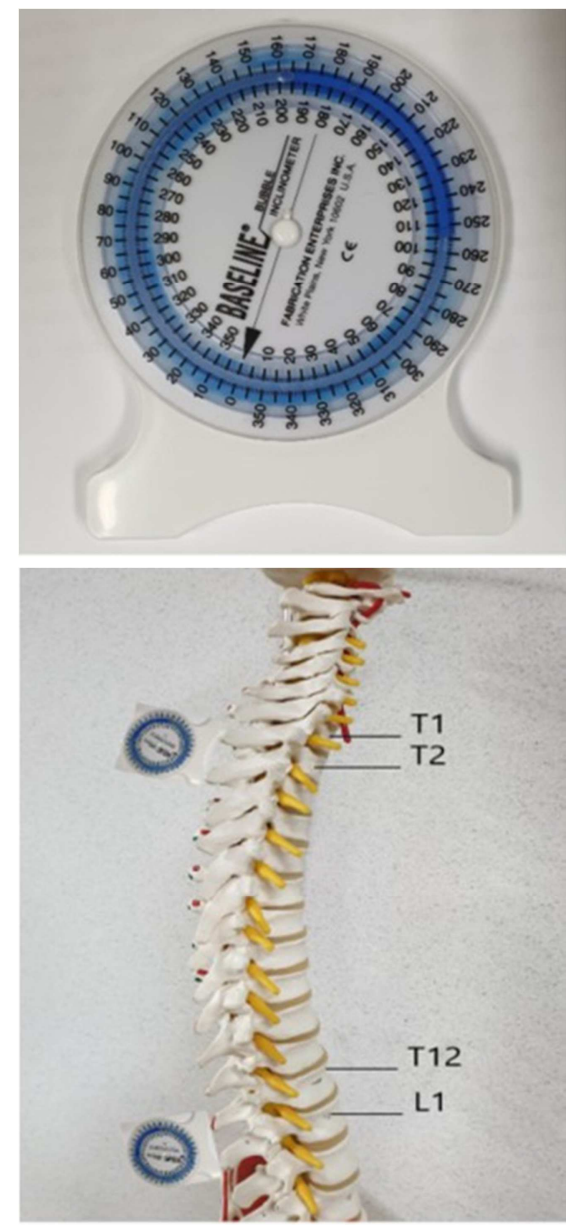

Fig 1. Inclinometers for mesure of kyphosis angle. The kyphosis angle was calculated by the summation of the angle recorded by the two inclinometers.

To take static measures of balance by Romberg open/ closed eyes test, the participant was asked to stand on the measuring instrument and look upward at a 15 degree angle, and to keep the front part of both feet in a posture rotating externally at about 30 degrees, before measuring the migration area of the center of pressure for 30 seconds (Park, 2017; Kim et al., 2014). The dynamic balance was measured by placing four measuring instruments on the auxiliary machine with four springs in the same posture used to measure the static balance (Fig. 2).

\section{Data analysis}

The data collected for this study was analyzed using SPSS 18.0 for Windows. Also, repeated one-way ANOVA was
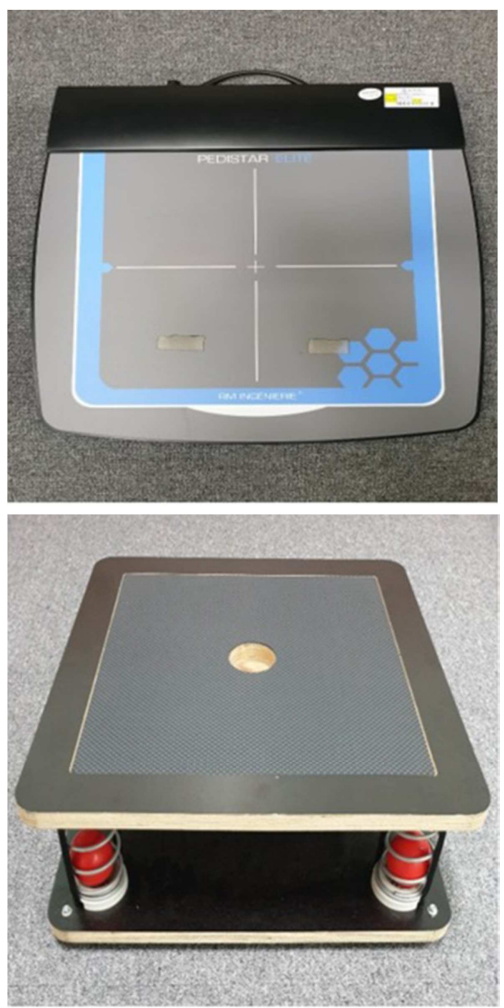

Fig 2. The Biorescue (RM Ingenierie, France), a instrument for measure balance ability. Biorescue is designed to measure the movement of the body's center of pressure (COP) at a point where the gravity influences the center of gravity (COG) of the body or object in order to assess the migration area $\left(\mathrm{mm}^{2}\right)$ while the movement is taking place.

performed to compare the effects achieved during 3 and 6 weeks after the application, respectively, in the 6-week application of the joint mobilization following the pre-testing, on changes to the angle of thoracic vertebrae and to the dynamic balance. The study also utilized the tests on within-subject effects with regard to sequential points of time for withinsubject variables. The significance level $(\alpha)$ was set at .05 .

\section{RESULTS}

\section{Comparing the kyphosis angle before and after the joint mobilization intervention}

The kyphosis angle based on the period during which joint mobilization is applied showed a significant decrease $(P$ $<.05)$ (Table 1). Moreover, through comparing the tests on within-subject effects of the kyphosis angle, a statistically sig- 
nificant difference was found in the application periods: before the intervention until 3 weeks after the intervention, and from 3 to 6 weeks after the intervention $(P<.05)$ (Table 2).

\section{Romberg test}

Static balance - eyes open: No statistically significant difference was found in changes to the movement area of the body's center of pressure based on the application period of the joint mobilization when the participant opened their eyes in a state of static balance $(P>.05)$ (Table 3$)$.

In addition, no statistically significant difference was seen when comparing the tests on within-subject effects of changes to the movement area of the body's center of pressure $(P>.05)$ (Table 4).

Static balance - eyes close: No statistically significant dif-

Table 1. Changes in the angle of thoracic kyphosis according to the period of TM

\begin{tabular}{|c|c|c|c|c|c|}
\hline & \multicolumn{3}{|c|}{ mean $\pm \mathrm{SD}$} & \multirow{2}{*}{$F$} & \multirow{2}{*}{$P$} \\
\hline & Pre & Post 3 weeks & Post 6 weeks & & \\
\hline $\mathrm{KA}\left({ }^{\circ}\right)$ & $48.11 \pm 8.14$ & $45.00 \pm 5.86$ & $42.11 \pm 4.98$ & 33.12 & $0.00^{*}$ \\
\hline
\end{tabular}

$P<.05$, TM: thoracic mobilization, KA: kyphosis angle

Table 2. Test for the angle of the kyphosis thoracic according to the period of TM

\begin{tabular}{lllc}
\hline \multicolumn{1}{c}{ Period } & MD & $F$ & $P$ \\
\hline Pre - Post 3 weeks & -3.11 & 18.77 & $0.00^{*}$ \\
Post 3 weeks - Post 6 weeks & -6.00 & 67.60 & $0.00^{*}$ \\
\hline
\end{tabular}

$P<.05$, TM: thoracic mobilization, MD: mean difference

Table 3. Variation of COP moving area of the body according to the period of TM in static balance in EO state

\begin{tabular}{|c|c|c|c|c|c|}
\hline & \multicolumn{3}{|c|}{ mean $\pm \mathrm{SD}$} & \multirow{2}{*}{$F$} & \multirow{2}{*}{$P$} \\
\hline & Pre & Post 3 weeks & Post 6 weeks & & \\
\hline $\mathrm{AM}\left(\mathrm{mm}^{2}\right)$ & $102.96 \pm 176.16$ & $80.04 \pm 123.66$ & $28.33 \pm 30.84$ & 1.48 & 0.26 \\
\hline
\end{tabular}

$P<.05$, TM: thoracic mobilization, COP: center of pressure, EO: eyes open, AM: area moving,

Table 4. Test for the COP moving area of the body according to the period of TM in static balance in EO state

\begin{tabular}{lccc}
\hline \multicolumn{1}{c}{ Period } & MD & $F$ & $P$ \\
\hline Pre - Post 3 weeks & -22.93 & 2.88 & 0.11 \\
Post 3 weeks - Post 6 weeks & -74.63 & 2.98 & 0.10 \\
\hline
\end{tabular}

$P<.05$, TM: thoracic mobilization, COP: center of pressure, EO: eyes open, MD: mean difference

Table 5. Variation of COP moving area of the body according to the period of TM in static balance in EC state

\begin{tabular}{|c|c|c|c|c|c|}
\hline & \multicolumn{3}{|c|}{ mean $\pm \mathrm{SD}$} & \multirow{2}{*}{$F$} & \multirow{2}{*}{$P$} \\
\hline & Pre & Post 3 weeks & Post 6 weeks & & \\
\hline $\mathrm{AM}\left(\mathrm{mm}^{2}\right)$ & $101.15 \pm 165.32$ & $72.30 \pm 87.81$ & $31.59 \pm 41.61$ & 1.52 & 0.25 \\
\hline
\end{tabular}

$P<.05$, TM: thoracic mobilization, COP: center of pressure, EC: eyes close, AM: area moving 
ference was found in changes to the movement area of the body's center of pressure based on the period of application of the joint mobilization when the participant closed their eyes in a state of static balance $(P>.05)$ (Table 5).

No statistically significant difference was seen when the tests on within-subject effects of changes to the movement area of the body's center of pressure were compared $(P$ $>$.05) (Table 6).
Dynamic balance - eyes open: A statistically significant difference was found in changes to the movement area of the body's center of pressure based on the application period of the joint mobilization when the participant opened their eyes in a state of dynamic balance $(P<.05)$ (Table 7$)$.

A statistically significant difference was also found as a result of comparing the tests on within-subject effects of changes to the movement area of the body's center of pres-

Table 6. Test for the COP moving area of the body according to the period of TM in static balance in EC state

\begin{tabular}{lccc}
\hline \multicolumn{1}{c}{ Period } & MD & $F$ & $P$ \\
\hline Pre - Post 3 weeks & -28.85 & 1.88 & 0.19 \\
Post 3 weeks - Post 6 weeks & -69.56 & 3.02 & 0.10 \\
\hline
\end{tabular}

$P<.05$, TM: thoracic mobilization, COP: center of pressure, EC: eyes close, MD: mean difference

Table 7. Variation of COP moving area of the body according to the period of TM in dynamic balance in EO state

\begin{tabular}{|c|c|c|c|c|c|}
\hline & \multicolumn{3}{|c|}{ mean \pm SD } & \multirow{2}{*}{$F$} & \multirow{2}{*}{$P$} \\
\hline & Pre & Post 3 weeks & Post 6 weeks & & \\
\hline $\mathrm{AM}\left(\mathrm{mm}^{2}\right)$ & $198.15 \pm 22.71$ & $187.59 \pm 18.261$ & $113.52 \pm 11.94$ & 14.76 & 0.00 \\
\hline
\end{tabular}

Table 8. Test for the COP moving area of the body according to the period of TM in dynamic balance in EO state

\begin{tabular}{lccc}
\hline \multicolumn{1}{c}{ Period } & MD & $F$ & $P$ \\
\hline Pre - Post 3 weeks & -10.56 & 0.49 & 0.49 \\
Post 3 weeks - Post 6 weeks & -84.63 & 25.79 & $0.00^{*}$ \\
\hline
\end{tabular}

$P<.05$, TM: thoracic mobilization, COP: center of pressure, EO: eyes open, MD: mean difference

Table 9. Variation of COP moving area of the body according to the period of TM in dynamic balance in EC state

\begin{tabular}{|c|c|c|c|c|c|}
\hline & \multicolumn{3}{|c|}{ mean $\pm \mathrm{SD}$} & \multirow{2}{*}{$F$} & \multirow{2}{*}{$P$} \\
\hline & Pre & Post 3 weeks & Post 6 weeks & & \\
\hline $\mathrm{AM}\left(\mathrm{mm}^{2}\right)$ & $680.67 \pm 447.67$ & $431.11 \pm 173.01$ & $273.19 \pm 89.57$ & 16.00 & $0.00^{*}$ \\
\hline
\end{tabular}

$P<.05$, TM: thoracic mobilization, COP: center of pressure, EC: eyes close, AM: area moving

Table 10. Test for the COP moving area of the body according to the period of TM in dynamic balance in EC state

\begin{tabular}{lccc}
\hline \hline \multicolumn{1}{c}{ Period } & MD & $F$ & $P$ \\
\hline Pre - Post 3 weeks & -249.56 & 9.92 & $0.02^{*}$ \\
Post 3 weeks - Post 6 weeks & -407.48 & 32.94 & $0.00^{*}$ \\
\hline
\end{tabular}

$P<.05$, TM: thoracic mobilization, COP: center of pressure, EC: eyes close, MD: mean difference 
sure, during an application period ranging from 3 to 6 weeks after the intervention $(P<.05)$ (Table 8$)$.

Dynamic balance - eyes close: A statistically significant difference was found in changes to the movement area of the body's center of pressure based on the application period of joint mobilization when the participant closed their eyes in a state of dynamic balance $(P<.05)$ (Table 9$)$.

A statistically significant difference was also found as a result of comparing the tests on within-subject effects of changes to the movement area of the body's center of pressure, during the application periods ranging from 'before the intervention' until 3 weeks after the intervention, and from 3 to 6 weeks after the intervention $(P<.05)$ (Table 10).

\section{DISCUSSION}

As a person moves, the central nervous system, which consists of the brain and the spinal cord, receives sensory information through the spinal cord. Then, the cerebrum plans movements and the cerebellum corrects the sensory information before providing control functions and commands for accurate, appropriate movements (Yun et al., 2013). As such, "balance" refers to an ability to maintain an upright posture, whereas posture stability can be defined as an individual's ability to maintain the body's center of gravity (COG) within the base of support (BOS) (Michael et al., 2013). In terms of the compensatory postural imbalance caused by the movement of COG, appropriate exercise strategies were observed for the head, the trunk and legs, with information input needed for maintaining balance in somatosense, vision and vestibular sense. It was found that the body keeps its balance by using the legs in support of the body on the ground and controlling strategic movements of ankle joint and hip joint (Mouchnino et al., 1998). The vertebral canal is adjacent to the central and peripheral nervous systems and the thoracic vertebrae are located between the cervical vertebrae and lumbar vertebrae (Kim, 2015). The variation of the kyphosis angle is closely correlated with leading to the flexion of the cervical vertebrae (Szeto et al., 2002). The muscle tone of nearby muscles to improve the stability of the spinal cord by means of the flexion of the cervical vertebrae leads to reducing proprioceptive input signals from the cervical vertebrae (Cheng et al., 2010).

In the Kaltenborn-Evjenth concept for patients with neck pain, the study conducted an experiment with two groups a group of seven participants on which joint mobilization was applied to the upper thoracic vertebrae and a group of another seven that underwent conservative physical therapy. According to the experiment, the group with joint mobilization showed a significant increase of the range of motion in 3 or 4 vertebral segments depending on the period, with no noticeable difference found in the group with conservative physical therapy (Jun, 2012). In this study, a significant difference was also found in the kyphosis angle in the comparison - before the intervention, 3 weeks after the intervention and 6 weeks after the intervention - depending on the application period of the joint mobilization.

It was found that a comparison was made for two groups of patients with chronic back pain - the group with joint mobilization applied to their thoracic vertebrae with limited mobility following a lumbar stabilization exercise and the lumbar stabilization exercise group. The comparison showed that both groups experienced, through the two treatment techniques, a pain reduction and improvements in somesthetic sense of proprioception (Yang and Kim, 2015). It was asserted that the reduction of kyphosis angle causes a change in the position of the cervical vertebrae, influencing postural alignment (Moon et al., 2014).

Postural balance is the ability to maintain efficient muscle tone, joint flexibility, muscular strength and endurance in combination with the nerve system and musculoskeletal system in order to adapt to changes in one's surroundings. To maintain these conditions, postural balance is the process in which the central nervous system integrates the information collected through vision, somesthetic sense, proprioception, cutaneous sensation, joint receptors and vestibular sense, in order to keep the body in balance. Of the components of sensory systems to keep the body in balance, proprioception in somesthesis is kept in balance and correlated, while the vision and vestibular senses serve as an auxiliary factor (Lee et al., 2002; Sturnieks et al., 2008; Carli et al., 2010). In a study concerning the proprioception of knee joints based on visual information involving eye closure and opening 
and the difference in thickness of the insole, it was found that a significant level of mean error was measured when the participant opened his/her eyes; however, a reduced error value was shown when eyes were closed (Kim et al., 2005). This can be understood as a static situation in which there are no motions.

For an experiment measuring the lumbar proprioception with the error value returning from the neutral posture through trunk flexion to the neutral posture, the participant wore an eye patch to block his/her vision and sat on a chair to exclude afferent input that comes from vestibular sense and feet. The experiment represented a reduction in proprioception due to pain, showing that the process of returning to neutral posture is maintained by the control through compensation not for the vision and vestibular sense but for proprioception (O'Sullivan et al., 2003). This is consistent with the fact that compensation by the vision and vestibular sense is unable to keep the body's posture in balance in the event of a problem associated with vestibular sense (Yang and Kim, 2015). In addition to this, research on a sensory weight was conducted (Oie et al., 2002). The sensory weight imposes an impact on body posture control by allowing other sensory organs to have an added weight when many sensory input organs of the body make changes to a specific sense. In this way, when visual information is reduced, somesthetic information is given a heavier weight, whereas when somesthetic information is reduced, more reliance is given to visual information to maintain posture control. However, in this study, the state of static balance does not belong to the environment sensitive to visual and somesthetic information that influence posture control, which therefore causes the sensory weight to remain unbiased. In a test for static balance while keeping the posture static, it was also considered that no significant difference is found when the participant closes their eyes and opens them.

When the participant opened their eyes in the dynamic balance, the balance ability was shown in a small area of movement prior to the experiment, also representing the same small area of movement even 3 and 6 respective weeks after the intervention. However, the movement area produced when the participant closed their eyes prior to the experiment was larger than when eyes were opened. But, as time went by, the kyphosis showed a reduction, with the movement area seeing a noticeable decrease as well. This appears to have had more focus on the sense of movement of the shaking board when eyes are closed, with influences on the stretch reflex at the ankles, somatosense and on the dynamic balance.

According to Carli et al. (2010), in the support posture for two legs, similar portions are found in vestibular sense, vision and proprioception input when the participant closed their eyes and opened them following the rehab training. On the other hand, keeping the posture with only one leg on the ground requires more proprioception input to the subject's leg while closing their eyes without any visual information. Moreover, the researchers found that the mechanism of spinal reflexes became revitalized in the leg contacting the ground while integrated with vestibular sense. It was reported by Dietz et al. (1991) that in an experiment which induces in the opposite direction that the head and trunk move to give change to vestibular sense, maintenance and control of the posture is performed by the vestibular organ and spinal reflexes. However, when the head moves faster, the response induced by vestibular sense was 10 times less than stretch reflexes by the displacement at the ankles. This verifies the mechanism of postural reflexes and control in terms of weight shift, and the sensory receptor in the joints and vertebrae provides information about the location of the body's center of gravity. This shows that the ability to control an exercise due to improved mobility of thoracic vertebrae also influences the balance ability. According to Hyung and Ha (2009), when manipulation was applied to the vertebral joints with reduced mobility to stimulate movements from front to back, muscles were relaxed by the stimulation on the tendon spindle caused by the muscle stretch, which increased the working range of movement to enhance the dynamic balance ability.

As seen above, the structural change to the kyphosis angle with the application of joint mobilization leads to proper alignment of the body, which has an impact on the balance due to changes to the muscle length, speed of movement and changes to joint locations. But, the static balance designed to provide static somesthetic information indicated that based on the application period, the structural change to the kyphosis angle did not present a significant difference 
in change to the movement area of the body's center of pressure. It appears that the kyphosis angle is changed over time - before the intervention, 3 and 6 respective weeks after the intervention - in a state of dynamic balance; information is smoothly delivered by the proper vertebral alignment; the displacement at the ankles on the auxiliary instrument causing the shaking makes stretch reflexes of ankle joints lead to spinal reflexes; postural reflex in weight shift and weighted information of somesthesis is dedicated to an increase in the balance control ability in the dynamic balance.

This study adopted selection criteria targeting people aged 20 or over without the symptoms of a disorder, and therefore may not be generally applied to all subjects. In addition, the researchers were faced with some limitations - such as insufficient control over living habits and failure to compare effects made by the intervention due to the lack of a control group. As such, future studies should address the limitations indicated above.

\section{ACKNOWLEDGEMENT}

None.

\section{CONFLICT OF INTEREST}

No conflicts of interest are involved in this manuscript.

\section{REFERENCES}

Carli P, Patrizi M, Pepe L, Cavaniglia G, Riva D, D'Ottavi LR. Postural control and risk of falling in bipodalic and monopodalic stabilometric tests of healthy subjects before, after visuo-proprioceptive vestibulo-postural rehabilitation and at 3 months thereafter: role of the proprioceptive system. Acta Otorhinolarygologica Italica. 2010. 30: 182-189.

Cheng CH, Wang JL, Lin JJ, Wang SF, Lin KH. Position accuracy and electromyographic responses during head reposition in young adults with chronic neck pain. Journal of Electromyography and Kinesiology. 2010. 20: 1014-1020.

Dietz V, Trippel M, Horstmann GA. Significance of Proprioceptive and Vestibulo - Spinal Reflexes in the Control of Stance and Gait. Advances in Psychology. 1991. 78: 37-52.

Geronimi M. Reproductibilité intra- et intersessions du test des limites destabilité sur plateforme podobarométrique. Neurophysiol Clinque. 2014. 44: 139.
Han AR, Jeong JI, Feng J, Kim YH. Kinematic Analysis of Thoraco-Lumbar Spine in Bad Postures During Daily Life. Transactions of the Korean Society of Mechanical Engineers. 2012. 36: 1105-1110.

Hwangbo PN. The Effects of Thoracic Joint Mobilization and Self Stretching Exercise on the Pulmonary Function of Patients with Chronic Neck Pain. Master's thesis. Daegu University, 2011.

Hyung IH, Ha MS. Effect of Immediate Dynamic Balance Ability of Chronic Low Back Pain on Maitland's Manual Therapy. The Korea Contents Society. 2009. 9: 209-213.

Jang HJ, Kim SY, Kim MJ. The Reliability and Relationships Between Thoracic Kyphosis, Postural Stiffness and Thoracic Rotation in Young Subjects. Korean Acad Orthop Man Physi Ther. 2012. 18: 49-55.

Joo YJ, Yoo JS. Survey on Special Education Teachers' Perceptions and Needs of Smart Devices Utilization. Journal of Special Education \& Rehabilitation Science. 2014. 53: 231-254.

Jun YW. The Effects of Upper Thoracic Joint Mobilization Technique using Kaltenborn-Evjenth concept on Cervicothoracic ROM and Pain in patients with Chronic Neck Pain. master's thesis. Korea Universit, 2012.

Jung HW, Shin WS, Kim DH, Park WH, Cha YY, Ko YS, Lee JH, Chung WS, Shin BC, Song YK, Go HY. The Study on Correlation between the Forward Head Posture and Spinal Alignment. Journal of Korean Medicine Rehabilitation. 2013. 23: 195-202.

Jung JD. The immediately Effects of Thoracolumbar Facet Joint Traction on the Spinal Mobility and Functional Ability of Patients with Chronic Low Back pain. Master's thesis. Konyang University, 2015.

Kaltenborn FM. The spine: Basic evaluation and mobilization techniques. 1th ed, Norway, Olaf norlis Bokhandel. 1993. pp.287.

Kim DK, Ko EH, Lee KS, Cynn HS. The Influences of Visual Imformation and Different Elevations of Medially Wedged Insoles on Knee Joint Proprioception in Healthy Persons. Phys Ther Korea. 2005. 12: 22-27.

Kim HG, Her JG, Ko JY. Effect of Horseback Riding Simulation Machine Training on Trunk Balance and Gait of Chronic Stroke Patient. J Phys Ther Sci. 2014. 26: 29-32.

Kim JG. The Role of Exercise for the Heath of Vertebral Column. Journal of the Korea Entertainment Industry Association. 2015. 9: 191-199.

Kim KI, Choi SY, Park DH. An A study on relationships between 
characteristics of smart usage and symptoms of MSDs. J. Korea Saf Manag Sci. 2016. 18: 119-129.

Kim KS. A study of Correlation between the Modified Spine Structure of Seat Pressure and Back Pressure and Spinal Health Status of University Students. Master's thesis. Woosong University, 2013.

Kim SW, Lee J, Lee BH. Effects of Degree of use of Smartphone on the Spinsl Health of Later Teens: Moderating Effect of Cognition Strength. Journal of Rehabilitation Research. 2018. 22: 213-230.

Kim SY, Jang HJ. Clinical Importance and Measurement in Thoracic Kyphosis. Korean Acad Orthop Man Physi Ther. 2012. 18: $1-10$.

Lee CS, Kang SS. Spino-Pelvic Parameters in Adult Spinal Deformities. Journal of the Korean Orthopaedic Association. 2016. 51: 9-29.

Lee HA. A Study on the Safety of Spinal Manipulation using Finite Element Method. Master's thesis. Korea University, 2010.

Lee HG, Cho JM, Bang JL, et al. A Study on the Utility of Sequential Whole Spine Lateral Standing Radiography for Dynamic Sagittal Imbalance Analysis. Journal of the Korean Society of Radiologocal Imaging Technology. 2011. 11: 1-16.

Lee WJ, Ju SB, Kwon HJ. The Effect of Proprioceptor Postural Therapy on Balance-Performing Ability. Korean Journal of Sports Science. 2002. 11: 503-509.

Lewis JS, Valentine RE. Clinical measurement of the thoracic kyphosis. A study of the intra-rater reliability in subjects with and without shoulder pain. BMC Musculoskelet Disord. 2010. 11: 1-7.

Michael R, Phil P, Nobuo T. Balance training for the Older Athlete. The International Journal of Sports Physical Therapy. 2013. 8: 517-530.

Mirbagheri SS, Rahmani-Rasa A, Farmani F, AminiP, Nikoo MR. Evaluating Kyphosis and Lordosis in Students by Using a Flexible Ruler and Their Relationship with Severity and Frequency of Thoracic and Lunbar pain. Asian Spine J. 2015. 9: 416-422.

Moon HH, Kim JH, Park YJ. The Effect of Improvement of the Spial Curvature, Cervical Pain and Neck Disability Index for Chronic Neck Patients with Kyphosis. Journal of Sport and Leisure Studies. 2014. 57: 925-933.

Mouchnino L, Mesure S, Lizee E, Landjerit B, Massion J. Is the spinal column a rigid or articulated axis during leg movement? Human Movement Science. 1998. 17: 289-306.

Nam HS. Effects of the Base of Support on Anticipatory Postural Adjustment and Postural Stability. Master's thesis. Daegu Catholic University, 2015.

Oie KS, Kiemel T, Jeka JJ. Multisensory fusion: simultaneous re-weighting of vision and touch for the control of human posture. Cognitive Brain Research. 2002. 14: 164-176.

O'Sullivan PB, Burnett A, Floyd AN, Gadsdon K, Logiudice J, Miller D, Quirke H. Lumbar repositioning deficit in a specific low back pain population. Spine. 2003. 28: 1074-1079.

Park HK. The Effects of Balance Training and Breathing Control Training on Balance, Respiration, Muscle Activity, and Shooting Record of Adolescent air Rifle Athletes. Doctor's Thesis. Daegu University. 2017.

Song JE, Kim SY, Jang HJ. A Comparison of the Effects of SelfMobilization and Strengthening Exercise of the Thoracic Region in Young Adults with Thoracic Hyperkyphosis. Korean Acad Orthop Man Physi Ther. 2013. 19: 11-18.

Sturnieks DL, St George R, Lord SR. Balance disorders in the elderly. neurophysiogie Clinique. 2008. 38: 467-478.

Sung YB, Lee JH, Park YH. Effedts of thoracic mobilization and manipulation on function and mental state in chronic lower back pain. J Phys Ther Sci. 2014. 26:1711-1714.

Szeto GP, Straker L, Raine S. A field comparison of neck and shoulder postures in symptomatic and asymptomatic office workers. Appl Ergon. 2002. 33: 75-84.

Yang JM, Kim SY. The Effect of Thoracic Joint Mobilization on Pain, Proprioception and Static Balance in Patients With Chronic Low Back Pain. Physical Therapy Korea. 2015. 22: 1-11.

Yun JE, Kim YH, Park JH. Lower limb's Proprioceptive Acuity with Position Matching Via Goal-directed Task. Journal of Coaching Development. 2013. 15: 129-139.

https://doi.org/10.15616/BSL.2019.25.2.149

Cite this article as: Jeong HJ, Kim BJ. The Effect of Thoracic Joint Mobilization on the Changes of the Thoracic Kyphosis Angle and Static and Dynamic Balance. Biomedical Science Letters. 2019. 25: 149-158. 